Now we go to the c.m. system where

$$
\begin{aligned}
& \mathbf{P}=0, \\
& \mathbf{p}=2 \mathbf{p}_{1}, \\
& \epsilon=\left|\mathbf{p}_{1}\right|^{2} / m,
\end{aligned}
$$

and

$$
\text { relative velocity }=|\mathbf{p}| / m=2\left|\mathbf{p}_{1}\right| / m \text {. }
$$

This is equivalent to a reduced-mass particle with momentum $\mathbf{p}_{1}$, so that

$$
\begin{aligned}
\epsilon & =\left|\mathbf{p}_{1}\right|^{2} / 2 \mu=\left|\mathbf{p}_{1}\right|^{2} / m \\
\text { velocity } & =\left|\mathbf{p}_{1}\right| / \mu=2\left|\mathbf{p}_{1}\right| / m .
\end{aligned}
$$

This situation can be represented symbolically as

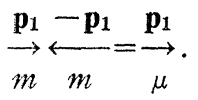

PHYSICAL REVIEW

Since we want to integrate over a "reduced particle" distribution, let us transform the integration variable from $\mathbf{p}$ to $\mathbf{p}_{1}$.

Using (A13),

Finally,

$$
(d \mathbf{p})=8\left(d \mathbf{p}_{1}\right)
$$

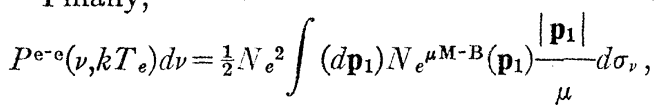

where $d \sigma_{\nu}$ is given by Eq. (1), where

$$
\epsilon_{1}=\left|\mathbf{p}_{1}\right|^{2} / 2 \mu \text {. }
$$

Integrating over angles, we obtain

$$
\begin{aligned}
& P^{\mathrm{e}-\mathrm{e}}\left(\nu, k T_{e}\right) d \nu=\frac{(2 / \pi \mu)^{1 / 2} N_{e}^{2}}{\left(k T_{e}\right)^{3 / 2}} \\
& \quad \times \int_{h \nu}^{\infty} d \epsilon_{1} \epsilon_{1} e^{-\epsilon_{1} / k T} d \sigma_{\nu}\left(\epsilon_{1}\right) .
\end{aligned}
$$

\title{
Kinetic Theory for the Interpretation of Measurements on Fluctuations in Radiation Distributions in Finite, Inhomogeneous Systems*
}

\author{
R. J. Gelinas \\ Lawrence Radiation Laboratory, University of California, Livermore, California \\ AND \\ R. K. OsBorn \\ Department of Nuclear Engineering, University of Michigan, Ann Arbor, Michigan
}

(Received 19 December 1966; revised manuscript received 5 June 1967)

\begin{abstract}
A kinetic (transport) theory is presented for the first- and second-order (and, if necessary, higher) statistical moments of the number densities of the various particles and/or photons that describe the observable fluctuations in the radiation distribution from an emitting system. This treatment is particularly suitable for the analysis of finite, inhomogeneous systems that may be composed of detectors located outside of a radiating source. Because we are largely concerned with the utility of kinetic theory as a physical theory, considerable emphasis is placed upon an appropriate theoretical description of the actual observables of given experimental situations. The quantum Liouville equation is used to generate the coupled set of transport equations, and basic criteria for the applicability of transport and wave theories are discussed. Quantumstatistical effects are also quite naturally accounted for in cases where they are relevant. It is seen that fluctuation measurements are useful for inferring information relevant to the dynamic interactions within a given system. Such measurements often enjoy the feature of being passive with respect to the interacting system of interest. To illustrate the use of this spatially dependent form of kinetic theory on a system emitting optical radiation, we consider an example that interprets a fluctuation measurement on the radiation emergent from a finite nondispersive blackbody. We conclude by discussing the problems of statistical coupling between the radiation field and detector atom distributions.
\end{abstract}

\section{INTRODUCTION}

$\mathrm{T}$ HE primary objective of this work is to present a transport theory of the multiplet densities of radiation distributions to facilitate the analysis of measurements of fluctuations in radiation fields in which spatial inhomogeneities play a significant role.

* Work performed under the auspices of the U. S. Atomic Energy Commission.
Our main concern will be for the development of the transport equations which describe the phase-space and time variation of physically interpretable singlet and doublet densities for the radiation system. A secondary objective is to apply these equations (as well as others needed for the description of detected particle densities) to an analysis of selected examples of fluctuation measurements on finite systems in which a consideration of spatially dependent effects is pertinent to a 
suitable interpretation of experimentally observable information.

The term "transport theory" is being used here generically, i.e., it is used in reference to those equations or systems of equations required for the description of averages of monomials of number operators in phase space. The average of an $n$ th-order monomial is often referred to as an $n$ th-order density. Common usage usually implies that the term "transport theory" refers to the mathematical description of a first-order (or singlet) density in phase space. Transport theory, therefore, is designed to describe energy transfer phenomena-to interpret those measurements which depend upon more or less discrete transfers of energy from one kind of a "particle" distribution to another. It is also designed to describe fluctuations in the number of such particles-both locally in phase space and otherwise. The notion of a particle here is purely intuitive, i.e., that of a highly localized concentration of mass and/or charge and/or energy, etc. The notion will be more or less useful and pictorial depending upon the circumstances in which it is employed. Thus transport theory conforms to and emphasizes the particulate view of complex (many-body) systems. Of course, the theory is not limited by the viewpoint; the formally exact (by postulate) equations descriptive of multiplet densities are valid whether or not the systems to which they are applied can be comprehended in intuitive terms.

All of this is in contrast to the "field theory" of complex systems. "Field theory" (or "wave theory") is a term used here to refer to those equations or systems of equations required for the description of averages of odd-order monomials (or linear combinations thereof for a given order) of creation and destruction operators. To our knowledge, no terminology has been invented for reference to the averages themselves; except for those of first order which are commonly referred to as fields. Since our motive here in discussing field theory at all is merely to provide comparison and contrast to transport theory, we recall in passing that Maxwell's equations are field equations in precisely the sense referred to above. The averages of certain Hermitian, linear, vector functions of creation and destruction operators define the real, vector fields which satisfy Maxwell's equations. For a wide range of situations, these fields are actually the observables of electrodynamics. A familiar instance of this situation is that class of experiments which are dominated by diffraction effects.

Conversely, experiments which measure energy transfer within, into, or out of systems to which the radiation is not too strongly coupled may lend themselves nicely to a transport-theoretic analysis. An extreme case for which this is expected to be true is that of $\gamma$-ray distributions in nuclear reactors. ${ }^{1}$ It might be

${ }^{1}$ R. J. Gelinas and R. K. Osborn, Nucl. Sci. Eng. 24, 184 (1966). noted that this spatially dependent kinetic theory may ultimately be of greatest value in stellar applications. For the present, however, measurements on such systems that would be appropriate for discussion from a kinetic theoretic point of view seem to be lacking. Others to which we feel the present theory is also applicable are cases in which the radiation emitted from a thermal optical source may be observed by one or more detectors placed outside of the source. The presence of certain types of optical equipment can also be accounted for. It should be recalled that measurements of numbers (or number densities) of particles are meaningfully interpreted by conventional transport theory only if the resolving times are long compared to characteristic lifetimes of states in systems of interest. It will also be seen that a transport approach is most appropriate only in instances where strong modal dependences of the electromagnetic field are absent.

In every instance, however, we are concerned with a careful consideration of the nature of the experimentally observed quantities (with due regard also for the dominant physical characteristics of the experimental setup in general) so that perhaps the most efficient and straightforward form of theoretical analysis will be suggested. Very often, though, one might expect to be dealing with systems where a clear division between particulate and wave features is not apparent from the distinguishing physical characteristics of the system. Such an example is an experiment that measures the ratio of the variance to mean or the power spectral density of detected particles in a system having a laser source..$^{2-4}$ However, since the main purpose of this paper is to present a tractable kinetic theory for finite, inhomogeneous systems, we intend to dwell only upon the simpler classes of experiments in which numbers of detected particles are observed from conventional, thermal radiation sources in the absence of strong modal properties of the electromagnetic fields and diffraction effects. The utility of this restricted consideration will become apparent in Sec. V, where it is seen that information relevant to dynamic parameters of the interacting system can be obtained in principle by passive measurements on the system over conveniently long time scales. The diagnostic potentialities of such measurements are of course significant. Finally it will also be seen that the approach taken herein is of sufficient generality to account for coupling effects between the radiation distribution and the distribution of detector atoms.

\section{DERIVATION OF THE EQUATIONS}

The arguments by which approximate transport equations have been deduced for the first- and second-

${ }^{2}$ J. A. Armstrong and Archibald W. Smith, Phys. Rev. Letters 14, 68 (1965).

3 D. E. McCumber, Phys. Rev. 141, 306 (1966).

${ }^{4}$ Charles Freed and Hermann A. Haus, Phys. Rev. 141, 287 (1966). 
order densities of the radiation system have been presented elsewhere ${ }^{5,6}$ in several analogous contexts, and hence require only minimal attention here. The singlet densities for particles of kind $A$ are defined by

$$
F_{1}{ }^{A}(\mathbf{X}, \mathbf{K}, a, t) \equiv\left[1 /(2 \pi)^{3}\right] \operatorname{Tr} \rho^{A}(\mathbf{X}, \mathbf{K}, a) D(t),
$$

and the doublet densities for particles of kinds $A$ and $B$ by

$$
\begin{array}{r}
F_{2}{ }^{A B}\left(\mathbf{X}, \mathbf{K}, a ; \mathbf{X}^{\prime}, \mathbf{K}^{\prime}, b ; t\right) \equiv\left[1 /(2 \pi)^{6}\right] \operatorname{Tr} \rho^{A}(\mathbf{X}, \mathbf{K}, a) \\
\times \rho^{B}\left(\mathbf{X}^{\prime}, \mathbf{K}^{\prime}, b\right) D(t) .
\end{array}
$$

The operators $\rho^{A}(\mathbf{X}, \mathbf{K}, a)$ are number operators whose eigenvalues in a diagonalizing representation represent the possible numbers of particles of kind $A$ in the phasespace hypercell centered at the point $(\mathbf{X}, \mathbf{K})$. The labels $a$ and $b$ specify the quantum numbers necessary to complete the description of the particle's state. They designate such things as polarization, spins, and internal states. All particles in a given phase cell are assigned the coordinates of the center of the cell, their momenta being given by $\mathbf{P}=h \mathbf{K}$. The volume of the cells is $(2 \pi)^{3}$. Evidently these phase points are discretely distributed; however, whenever appropriate, they will be assumed to be sufficiently dense to be regarded as a continuum. The quantity $D$ is the density operator for the system. The system is comprised of all of the particles of all kinds necessary for a model adequate to the interpretation of a given experiment.

The density operator is assumed to satisfy the Liouville equation given by

$$
\frac{\partial D(t)}{\partial t}=\frac{i}{h}[D, H]
$$

where $H$ is the Hamiltonian of the system. It is useful to write $H$ as

$$
H=\sum_{A} H^{A}+V
$$

In this case $H^{A}$ describes the kinetic energy of the $A$-type particle (this includes the contribution from the free photon field), and $V$ represents all other contributions to the energy of the system.

The first step in the present derivation of a transport equation is to display (generically)

$$
\frac{F(t+\tau)-F(t)}{\tau}=\frac{\partial F}{\partial t}\left[1+\frac{\tau}{2} \frac{\partial^{2} F / \partial t^{2}}{\partial F / \partial t}+\cdots\right] \simeq \frac{\partial F}{\partial t},
$$

for sufficiently small $\tau$ and for densities which do not vary too rapidly in time. For example, if $F(t) \sim e^{t / T}$, then the above approximation implies the neglect of a series of terms, the largest of which is $O(\tau / T)$ for $\tau / T<1$. An obvious lower limit for $\tau$ is effective interaction times; which, in the case of natural sources, will

${ }^{5}$ R. K. Osborn, Phys. Rev. 130, 2142 (1963).

${ }^{6}$ R. K. Osborn and M. Natelson, J. Nucl. Energy 19, 619 (1965). not likely exceed $10^{-10} \mathrm{sec}$. It is to be noted that this approximation (coarse graining in time) is a necessity and not merely a calculational trick, since it is meaningless to compare densities at two instants closer together than an interaction time.

It can then be shown $n^{5,6}$ that, in a representation which diagonalizes the number operator, the equation for the singlet density of the $A$-type particles can be written as

$$
\begin{aligned}
& {\left[\frac{\partial}{\partial t}+\mathbf{v}^{A} \cdot \nabla\right] F_{1^{A}}{ }^{A}(\mathbf{X}, \mathbf{K}, a ; t)} \\
& =\frac{1}{(2 \pi)^{3}} \sum_{n n^{\prime}}\left[\rho_{n^{\prime} n^{A}}{ }^{A}(\mathbf{X}, \mathbf{K}, a)-\rho_{n n}{ }^{A}(\mathbf{X}, \mathbf{K}, a)\right] T_{n^{\prime} n} D_{n n}(t) \\
& \quad+\text { terms off-diagonal in } D(t)
\end{aligned}
$$

We have introduced $\mathbf{v}^{A}$ to represent the velocity of the $A$-type particles. Note that $\mathbf{v}^{A} \equiv \hbar \mathbf{K} / m_{A}$ when $A$ applies to an ordinary particle, and $m_{A}$ is the mass of the particle. When $A$ applies to photons, $\mathbf{v}^{A} \equiv c \boldsymbol{\Omega}$ where $c=3 \times 10^{10} \mathrm{~cm} / \mathrm{sec}$ for a nondispersive medium, and $\boldsymbol{\Omega}$ is the direction vector for the photon. $T_{n^{\prime} n}$ stands for the probability per unit time for a transition to occur between an initial state designated by $n$ and a final state designated by $n^{\prime}$. The summation over $n$ and $n^{\prime}$ includes all initial and final states. Expressions for $T_{n^{\prime} n}$ can be obtained by conventional perturbation techniques when both the initial and final states are stable. Damping theory is invoked to account for finite lifetime effects in appropriate circumstances. Many approximations are required to go from (1), (3), and (5) to (6) -all of which have been displayed explicitly, some interpreted qualitatively, but few estimated quantitatively. Since many of these considerations have been dealt with specifically in Refs. 5 and 6 , we proceed at this point to writing the generic doublet equation [neglecting terms proportional to off-diagonal elements of $D(t)$ henceforth] as

$$
\begin{array}{r}
{\left[\frac{\partial}{\partial t}+\mathbf{v}^{A} \cdot \boldsymbol{\nabla}+\mathbf{v}^{B} \cdot \nabla^{\prime}\right] F_{2}{ }^{A B}\left(\mathbf{X}, \mathbf{K}, a ; \mathbf{X}^{\prime}, \mathbf{K}^{\prime}, b ; t\right)} \\
=\frac{1}{(2 \pi)^{6}} \sum_{n^{\prime} n} T_{n^{\prime} n}\left[\rho_{n^{\prime} n^{\prime}}{ }^{A}(\mathbf{X}, \mathbf{K}, a) \rho_{n^{\prime} n^{\prime}}{ }^{B}\left(\mathbf{X}^{\prime}, \mathbf{K}^{\prime}, b\right)\right. \\
\left.-\rho_{n n}{ }^{A}(\mathbf{X}, \mathbf{K}, a) \rho_{n n}{ }^{B}\left(\mathbf{X}^{\prime}, \mathbf{K}^{\prime}, b\right)\right] D_{n n}(t) .
\end{array}
$$

Note that the gradients which appear in the equations at this point are symbolic and have the meaning of a finite difference in the density of interest at two adjacent cells in configuration space, divided by the linear dimension of the cells. They will take on the usual meaning of gradients when we pass to the continuum for densely spaced points.

Further specialization of these equations requires considering individually the relevant interactions on the 
right-hand side of the balance equations. To do this it is useful to restrict our attention to the dependence of the transition probability per unit time, $T_{n^{\prime} n}$, upon occupation numbers only. In the cases that we consider, the binary collision assumption is made so that $T_{n^{\prime} n}$ bears a proportionality to $\rho_{n n}$ for each annihilation process as well as a proportionality to $\left[1 \pm \rho_{n n}\right]$ (where the plus and minus signs go with bosons and fermions, respectively) for each creation process. If, in the creation of a particle, $\rho_{n n}$ is not negligible compared to unity, the effect of quantum statistics will be observed.

In systems that we will consider, the dominant processes are the production of photons by source atom transitions, their absorption by detection processes, and perhaps some self-absorption by the source. In instances where optical equipment such as a beam splitter or a polarizer is present, terms to account for photon scattering are included in addition to the above processes. If then in writing the equations for the densities of particles that describe the system (in this case photons and photoelectrons), we use the superscript $A$ to represent the set composed only of photons and of detected particles (photoelectrons) and let $A^{\prime}$ include all other kinds of particles, we have for the set of singlet equations after summing over photon polarizations and photoelectron spins:

$$
\begin{aligned}
& {\left[\frac{\partial}{\partial t}+\mathbf{v}^{A} \cdot \boldsymbol{\nabla}\right] F_{1}{ }^{A}(\mathbf{X}, \mathbf{K}, t)=\frac{1}{(2 \pi)^{3}} \sum_{n, a} D_{n n}(t)\left\{\left[1 \pm \rho_{n n}{ }^{A}(\mathbf{X}, \mathbf{K}, a)\right] \sum_{\substack{A^{\prime}, \mathbf{K}_{1}, a_{1} \\
\mathbf{K}_{2}, a_{2}, \mathbf{K}^{\prime}, a^{\prime \prime}}} \rho_{n n^{\prime \prime}}\left(\mathbf{X}, \mathbf{K}_{1}, a_{1}\right)\right.} \\
& \times\left[T_{e}\left(a, \mathbf{K} \mid \mathbf{K}_{\mathbf{1}}, a_{1} ; \mathbf{K}_{2}, a_{2}\right)+\rho_{n n}{ }^{A}\left(\mathbf{X}, \mathbf{K}^{\prime \prime}, a^{\prime \prime}\right) T_{\text {so }}\left(a, \mathbf{K}\left|\mathbf{K}_{\mathbf{1}}, a_{1} ; \mathbf{K}_{\mathbf{2}}, a_{2}\right| \mathbf{K}^{\prime \prime}, a^{\prime \prime}\right)\right] \\
& -\rho_{n n}{ }^{A}(\mathbf{X}, \mathbf{K}, a) \sum_{\substack{A \\
\mathbf{K}_{2,2}, \mathbf{K}_{1}, \mathbf{K}^{\prime \prime}, a^{\prime \prime}}} \rho_{n n} A^{A^{\prime}}\left(\mathbf{X}, \mathbf{K}_{\mathbf{1}}, a_{1}\right)\left[T_{a}\left(a, \mathbf{K} \mid \mathbf{K}_{\mathbf{1}}, a_{1} ; \mathbf{K}_{2}, a_{2}\right)\right. \\
& \left.\left.+\left(1 \pm \rho_{n n}{ }^{A}\left(\mathbf{X}, \mathbf{K}^{\prime \prime}, a^{\prime \prime}\right)\right) T_{\mathrm{sc}}\left(a^{\prime \prime}, \mathbf{K}^{\prime \prime}\left|\mathbf{K}_{1}, a_{1} ; \mathbf{K}_{2}, a_{2}\right| \mathbf{K}, a\right)\right]\right\} .
\end{aligned}
$$

Note that

$$
F_{1}{ }^{A}(\mathbf{X}, \mathbf{K}, t)=\sum_{a} F_{1}{ }^{A}(\mathbf{X}, \mathbf{K}, a, t)
$$

and

$$
\rho^{A}(\mathbf{X}, \mathbf{K})=\sum_{a} \rho^{A}(\mathbf{X}, \mathbf{K}, a) .
$$

The reader is also alerted to a specialization that is implicit in Eq. (8), and subsequently in Eq. (10), as they are written here. That is, in the systems considered in this paper we neglect photon scattering within the emitting source but include photon scattering for cases in which photons may scatter in an exterior region (from the source) prior to detection. In addition, the cases that we consider are such that the radiation is not too strongly coupled to the source system, and the source atom distribution is assumed to be known. The question of photons scattering with other photons is not of interest for our purposes, and the process is accordingly neglected herein. In this regard, the effect of interactions of detected particles with their own kind, and with atoms of the photo-cathode materials, are inferred by introducing (at a later point) a factor that suitably accounts for the macroscopic characteristics of the detection system. In these inhomogeneous systems there is no direct interaction of particles in the detection part of the system, for instance, with the optical equipment or with the radiation source atoms. Corresponding statements can be made regarding the source atoms and optical components. The reemission, by the detector atoms, of radiation with the properties of the source radiation is assumed to be negligible. In the above equations the quantity $T_{e}\left(a, \mathbf{K} \mid \mathbf{K}_{\mathbf{1}}, a_{1} ; \mathbf{K}_{2}, a_{2}\right)$ is the transition probability per unit time per atom for the emission of an $A$-type particle with wave vector $\mathbf{K}$ and quantum state label $a$. The state of the atom prior to undergoing a transition is designated by $K_{1}, a_{1}$. The wave vector associated with the atom in its initial state is $\mathbf{K}_{1}$, and $a_{1}$ represents the initial internal state of the atom. Then $\mathbf{K}_{2}, a_{2}$ are defined analogously for final states of the atom (after a transition). The quantity $T_{a}\left(a, \mathbf{K} \mid \mathbf{K}_{1}, a_{1} ; \mathbf{K}_{2}, a_{2}\right)$ represents the transition probability per unit time per atom for the absorption of an $A$-type particle with wave vector $\mathbf{K}$ and quantum labels $a$. The initial and final states of the atom involved in the transition are designated by $\mathbf{K}_{1}, a_{1}$ and $\mathbf{K}_{2}, a_{2}$, respectively, as above. For the emission and absorption of photons by source atoms, $T_{e}$ and $T_{a}$ are matrix elements that go into each other upon interchanging the initial and final source atom states that are involved. When the detection process is considered, one finds that $T_{e}$ (for the emission of photoelectrons) is proportional to the photon occupation numbers; and the sum over initial states includes a summation over the variables of the photon operator. Since we are considering emission and/or absorption of single particles, only those final states for which $\left[\rho_{n^{\prime} n^{\prime}}{ }^{A}(\mathbf{X}, \mathbf{K}, a)-\rho_{n n}{ }^{A}(\mathbf{X}, \mathbf{K}, a)\right]$ is plus or minus one, respectively, are contributors from the summation over all final states. The quantity $T_{\mathrm{sc}}\left(a, \mathbf{K}\left|\mathbf{K}_{\mathbf{1}}, a_{1} ; \mathbf{K}_{2}, a_{2}\right| \mathbf{K}^{\prime \prime}, a^{\prime \prime}\right)$ is the transition probability per unit time per atom for an $A$-type particle with initial wave vector $\mathbf{K}^{\prime \prime}$ and quantum state label $a^{\prime \prime}$ to undergo a scattering interaction resulting in the $A$-type particle having a final wave vector $\mathbf{K}$ and quantum label $a$. The initial and final states of the scattering atom are designated as above. The symmetry properties 
of $T_{\mathrm{sc}}\left(a, \mathbf{K}\left|\mathbf{K}_{\mathbf{1}}, a_{1} ; \mathbf{K}_{2}, a_{2}\right| \mathbf{K}^{\prime \prime}, a^{\prime \prime}\right)$ and $T_{\mathrm{sc}}\left(a^{\prime \prime}, \mathbf{K}^{\prime \prime} \mid \mathbf{K}_{\mathbf{1}}, a_{1}\right.$; $\left.\mathbf{K}_{2}, a_{2} \mid \mathbf{K}, a\right)$ are apparent, and the stimulated emission of particles other than photons is taken to be negligible.
In like manner as in (8) we can write the set of doublet equations (once again restricting $A$ and $B$ to photons and detected particles) as

$$
\begin{aligned}
& {\left[\frac{\partial}{\partial t}+\mathbf{v}^{A} \cdot \nabla+\mathbf{v}^{B} \cdot \nabla^{\prime}\right] F_{2}^{A B}\left(\mathbf{X}, \mathbf{K} ; \mathbf{X}^{\prime}, \mathbf{K}^{\prime} ; t\right)} \\
& =\frac{1}{(2 \pi)^{6}} \sum_{n, a, b} D_{n n}(t)\left[\left[1 \pm \rho_{n n}{ }^{A}(\mathbf{X}, \mathbf{K}, a)\right] \rho_{n n}{ }^{B}\left(\mathbf{X}^{\prime}, \mathbf{K}^{\prime}, b\right) \sum_{\substack{A \\
\mathbf{K}_{2, a_{2}, \mathbf{K}^{\prime \prime}, a^{\prime \prime}}}} \rho_{n n}{ }^{A^{\prime}}\left(\mathbf{X}, \mathbf{K}_{\mathbf{1}}, a_{1}\right)\right. \\
& \times\left[T_{e}\left(a, \mathbf{K} \mid \mathbf{K}_{\mathbf{1}}, a_{1} ; \mathbf{K}_{2}, a_{2}\right)+\rho_{n n}{ }^{A}\left(\mathbf{X}, \mathbf{K}^{\prime \prime}, a^{\prime \prime}\right) T_{\mathrm{sc}}\left(a, \mathbf{K}\left|\mathbf{K}_{\mathbf{1}}, a_{1} ; \mathbf{K}_{2}, a_{2}\right| \mathbf{K}^{\prime \prime}, a^{\prime \prime}\right)\right]+\left[1 \pm \rho_{n n}{ }^{B}\left(\mathbf{X}^{\prime}, \mathbf{K}^{\prime}, b\right)\right] \\
& \times \rho_{n n}{ }^{A}(\mathbf{X}, \mathbf{K}, a) \sum_{\substack{\mathbf{K}_{2}, b_{2}, \mathbf{K}_{1}, \mathbf{L}^{\prime \prime}, b^{\prime \prime}}} \rho_{n n}^{B^{\prime}}\left(\mathbf{X}^{\prime}, \mathbf{K}_{\mathbf{1}}, b_{1}\right)\left[T_{e}\left(b, \mathbf{K}^{\prime} \mid \mathbf{K}_{1}, b_{1} ; \mathbf{K}_{2}, b_{2}\right)+\rho_{n n}{ }^{B}\left(\mathbf{X}^{\prime}, \mathbf{K}^{\prime \prime}, b^{\prime \prime}\right) T_{\mathrm{sc}}\left(b, \mathbf{K}^{\prime}\left|\mathbf{K}_{1}, b_{1} ; \mathbf{K}_{2}, b_{2}\right| \mathbf{K}^{\prime \prime}, b^{\prime \prime}\right)\right] \\
& -\rho_{n n}{ }^{A}(\mathbf{X}, \mathbf{K}, a) \rho_{n n}{ }^{B}\left(\mathbf{X}^{\prime}, \mathbf{K}^{\prime}, b\right)\left(\sum _ { \substack { A _ { 2 } ^ { \prime } , \mathbf { K } _ { 1 } , a _ { 1 } \\
\mathbf { K } _ { 2 , } , \mathbf { K } ^ { \prime \prime } , a ^ { \prime \prime } } } \rho _ { n n } A ^ { \prime } ( \mathbf { X } , \mathbf { K } _ { \mathbf { 1 } } , a _ { 1 } ) \left\{T_{a}\left(a, \mathbf{K} \mid \mathbf{K}_{\mathbf{1}}, a_{1} ; \mathbf{K}_{2}, a_{2}\right)+\left[1 \pm \rho_{n n}{ }^{A}\left(\mathbf{X}, \mathbf{K}^{\prime \prime}, a^{\prime \prime}\right)\right]\right.\right.
\end{aligned}
$$

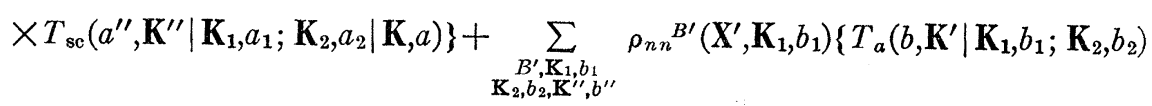

$$
\begin{aligned}
& \left.\left.+\left[1 \pm \rho_{n n}{ }^{B}\left(\mathbf{X}^{\prime}, \mathbf{K}^{\prime \prime}, b^{\prime \prime}\right)\right] T_{\mathrm{sc}}\left(b^{\prime \prime}, \mathbf{K}^{\prime \prime}\left|\mathbf{K}_{\mathbf{1}}, b_{1} ; \mathbf{K}_{2}, b_{2}\right| \mathbf{K}, b\right)\right\}\right)+(2 \pi)^{3} \delta\left(\mathbf{X}-\mathbf{X}^{\prime}\right)\left(\delta\left(\mathbf{K}-\mathbf{K}^{\prime}\right) \delta_{A B} \delta_{a b}\right. \\
& \times\left\{[ 1 \pm \rho _ { n n } { } ^ { A } ( \mathbf { X } , \mathbf { K } , a ) ] \sum _ { \substack { A ^ { \prime } , \mathbf { K } _ { 1 , } , a _ { 1 } ^ { \prime } \\
\mathbf { K } _ { 2 , a } , \mathbf { K } ^ { \prime \prime } , a ^ { \prime \prime } } } \rho _ { n n } { } ^ { A ^ { \prime } } ( \mathbf { X } , \mathbf { K } _ { 1 } , a _ { 1 } ) \left[T_{e}\left(a, \mathbf{K} \mid \mathbf{K}_{1}, a_{1} ; \mathbf{K}_{2}, a_{2}\right)+\rho_{n n}{ }^{A}\left(\mathbf{X}, \mathbf{K}^{\prime \prime}, a^{\prime \prime}\right)\right.\right. \\
& \left.\times T_{\mathrm{sc}}\left(a, \mathbf{K}\left|\mathbf{K}_{\mathbf{1}}, a_{1} ; \mathbf{K}_{2}, a_{2}\right| \mathbf{K}^{\prime \prime}, a^{\prime \prime}\right)\right]+\rho_{n n}{ }^{A}(\mathbf{X}, \mathbf{K}, a) \sum_{\substack{A^{\prime}, \mathbf{K}_{1}, a_{1} \\
\mathbf{K}_{2,}, a_{2}, \mathbf{\prime}^{\prime \prime}, a^{\prime \prime}}} \rho_{n n^{A^{\prime}}}\left(\mathbf{X}, \mathbf{K}_{\mathbf{1}}, a_{1}\right) \\
& \left.\times\left(T_{a}\left(a, \mathbf{K} \mid \mathbf{K}_{\mathbf{1}}, a_{1} ; \mathbf{K}_{2}, a_{2}\right)+\left[1 \pm \rho_{n n}{ }^{A}\left(\mathbf{X}, \mathbf{K}^{\prime \prime}, a^{\prime \prime}\right)\right] T_{\mathrm{sc}}\left(a^{\prime \prime}, \mathbf{K}^{\prime \prime}\left|K_{\mathbf{1}}, a_{1} ; \mathbf{K}_{2}, a_{2}\right| \mathbf{K}, a\right)\right)\right\} \\
& -\delta_{A B} \delta_{a b}\left\{\rho_{n n}{ }^{A}(\mathbf{X}, \mathbf{K}, a) \sum_{\substack{A^{\prime}, \mathbf{K}_{1}, a_{1} \\
\mathbf{K}_{2}, a_{2}}} \rho_{n n}{ }^{A^{\prime}}\left(\mathbf{X}, \mathbf{K}_{1}, a_{1}\right)\left[1 \pm \rho_{n n}{ }^{A}\left(\mathbf{X}, \mathbf{K}^{\prime}, a\right)\right] T_{\mathrm{sc}}\left(a, \mathbf{K}^{\prime}\left|\mathbf{K}_{1}, a_{1} ; \mathbf{K}_{2}, a_{2}\right| \mathbf{K}, a\right)\right. \\
& +\rho_{n n}{ }^{A}\left(\mathbf{X}, \mathbf{K}^{\prime}, a\right) \sum_{\substack{A^{\prime}, \mathbf{K}_{1, a}, a_{1} \\
\mathbf{K}_{2,, a_{2}}}} \rho_{n n} A^{A^{\prime}}\left(\mathbf{X}, \mathbf{K}_{\mathbf{1}}, a_{1}\right)\left[1 \pm \rho_{n n}{ }^{A}(\mathbf{X}, \mathbf{K}, a) T_{\mathrm{sc}}\left(a, \mathbf{K}\left|\mathbf{K}_{\mathbf{1}}, a_{1} ; \mathbf{K}_{2}, a_{2}\right| \mathbf{K}^{\prime}, a\right)\right\} \\
& \left.\left.-\left(1-\delta_{A B}\right) \rho_{n n}{ }^{A}(\mathbf{X}, \mathbf{K}, a) R_{A B}\left(\mathbf{K} \rightarrow \mathbf{K}^{\prime}\right)\right)\right] .
\end{aligned}
$$

The quantity $R_{A B}\left(\mathbf{K} \rightarrow \mathbf{K}^{\prime}\right)$ is the probability per unit time that a particle of type $A$ with wave vector $\mathbf{K}$ is absorbed and a $B$-type particle is subsequently reemitted with wave vector $\mathbf{K}^{\prime}$. This applies in particular to the emission of photoelectrons from a photon detection event, such that $\rho_{n n}{ }^{A}(\mathbf{X}, \mathbf{K}, a) R_{A B}\left(\mathbf{K} \rightarrow \mathbf{K}^{\prime}\right)$ is given by

$$
\rho_{n n}^{R}\left(\mathbf{X}, \mathbf{K}_{\mathbf{1}}, a\right) \sum_{\substack{\mathbf{K}_{1, a 1}, \rho_{1} \\ \mathbf{K}_{2, a 2}}} \rho_{n}^{A D}\left(\mathbf{X}, \mathbf{K}_{\mathbf{1}}, a_{1}\right) T_{\epsilon}\left(\mathbf{K}^{\prime}, b \mid \mathbf{K}_{\mathbf{1}}, a_{1} ; \mathbf{K}_{2}, a_{2}\right),
$$

where the superscript $A_{D}$ represents detector atoms, and $R$ denotes photons [in $T_{e}$ we will neglect the photoelectron occupation number $\rho_{n n}{ }^{D}\left(\mathbf{X}, \mathbf{K}^{\prime}, b\right)$ compared to unity]. The above equations have been written in a more or less generalized form (except for the specializations that were indicated above) in hope that they may be useful for more general applications in the future. The specializations invoked are for the sake of temporary convenience only, and it is clear in principle that strictly generic forms for these equations can be written with only little more effort but somewhat more space.

\section{EXPERIMENTAL OBSERVABLES OF A FLUCTUATION MEASUREMENT}

The very notion of fluctuations requires the consideration of at least second-order statistical quantities. In considering energy transfer measurements, such as the detection of radiation by photocathodes, it is convenient to work in terms of the doublet (and singlet) densities of the detected particles. It is easily shown that the mean-square value of the fluctuations in the number of detection events in the time interval $T$ from a single detector, located at $\mathbf{X}$, may be expressed as

$$
\begin{aligned}
\left\langle\left(\Delta N_{T}\right)^{2}\right\rangle & =\left\langle\left(N_{T}{ }^{D}\right)^{2}\right\rangle-\left\langle N_{T^{D}}\right\rangle^{2} \\
& \equiv \int_{\{\text {D.v. }\}} d^{\mathbf{6} X} V^{D D}(\mathbf{X}, \mathbf{X}, T)=V^{D D}(T) .
\end{aligned}
$$


The angular brackets denote expectation values, so that for instance $\left\langle N_{T}{ }^{D}\right\rangle$ is the mean number of detection events accumulating in a time interval of length $T$; and $\{D . V$.$\} represents the detector volume. The variance$ of detected particles, $V^{D D}(\mathbf{X}, \mathbf{X}, T)$ is defined by

$$
V^{D D}(\mathbf{X}, \mathbf{X}, T) \equiv f_{2}{ }^{D D}(\mathbf{X}, \mathbf{X}, T)-\left[f_{\mathbf{1}}{ }^{D}(\mathbf{X}, T)\right]^{2} .
$$

The quantities on the right-hand side of Eq. (12) can be obtained in practice by straightforward data analysis techniques from a suitable recording of the detection events. Thus the connection between theory and experiment is quite direct in this case. Note that a cross covariance of the detection events in a two-detector experiment is defined analogously (for detectors located at $\mathbf{X}_{1}$ and $\mathbf{X}_{2}$ ) as

$$
V^{D D}\left(\mathbf{X}_{1}, \mathbf{X}_{2}, T\right) \equiv f_{2}{ }^{D D}\left(\mathbf{X}_{1}, \mathbf{X}_{2}, T\right)-f_{1}{ }^{D}\left(\mathbf{X}_{1}, T\right) f_{1}{ }^{D}\left(\mathbf{X}_{2}, T\right) .
$$

Another useful quantity for fluctuation measurements is the generalized cross-correlation function $\phi(\tau)$ associated with the fluctuating currents from two detectors. It is defined as

$$
\phi(\tau)=\frac{1}{2}(1+P)\left\langle\Delta \mathscr{g}_{1}(t) \Delta \mathfrak{g}_{2}(t+\tau)\right\rangle,
$$

where $P$ is simply a permutation operator that interchanges 1 and 2. Note that, for currents from stationary systems, $\phi$ is a function of $\tau$ only. We have defined $\Delta \mathfrak{g}(t)$ in Eq. (14) as

$$
\Delta \mathfrak{g}(t)=\mathscr{g}(t)-\langle g(t)\rangle,
$$

where $g(t)$ is the instantaneous value of the output current. It is also noted that $\langle g(t)\rangle$ is simply the time rate of change of the expected number of detected particles in time $t$, or

$$
\langle g(t)\rangle=\partial f_{1}{ }^{D}(t) / \partial t=\text { constant }
$$

for stationary systems. It can then be shown ${ }^{7}$ that

$\phi(\tau)=\frac{1}{2} \int_{\{\text {D.v. }\}_{1}} d^{3} X_{1} \int_{\{\text {D.v. }\}_{2}} d^{3} X_{2} \frac{\partial^{2} V^{D D}\left(\mathbf{X}_{1}, \mathbf{X}_{2}, \tau\right)}{\partial \tau^{2}}$.

In the case of a single-detector experiment one lets $\{\text { D.V. }\}_{1}=\{\text { D.V. }\}_{2}=\{$ D.V. $\}$. The next step is to write (and subsequently solve) the set of equations for $f_{1}{ }^{D}(t)$, for $f_{2}{ }^{D D}(t)$, and for the other quantities (particularly cross-doublet densities for radiation and detected particles) that will be seen to couple these densities to the radiation distribution.

\section{FURTHER REDUCTION AND A METHOD OF SOLUTION OF THE COUPLED SET OF TRANSPORT EQUATIONS}

Referring back to Eqs. (8) and (10) and the attendant discussion there, we see that the emission and absorp-

${ }^{7}$ A. Papoulis, Probability, Random Variables, and Stochastic Processes (McGraw-Hill Book Company, Inc., New York, 1965). tion probabilities $T_{e}$ and $T_{a}$ are nonzero only for source atoms and detector atoms. Scattering terms will be included only to account for the interaction of radiation with selected pieces of optical equipment outside of the emitting source, and the photon occupation number is neglected in comparison to unity in the exterior region (to the source). Since photon scattering is otherwise neglected in our systems, $T_{\mathrm{se}}$ will usually be a very localized function in space. The distributions of the atoms comprising optical equipment, of the radiation source, and of the photocathode material are assumed to be known. Detected particles are simply recorded and their number merely accumulates with time. Finally we integrate photoelectron energies and directions over their entire range, neglect photoelectron streaming, and assume photon densities to be stationary. It is then a straightforward matter to write the reduced set of equations, starting with the photoelectrons (detected particles) $D$ as

$$
\begin{gathered}
\frac{\partial f_{1} D(\mathbf{X}, t)}{\partial t}=\Delta f_{1}{ }^{R}(\mathbf{X}) H(t) \\
\frac{\partial f_{2}{ }^{D D}\left(\mathbf{X}, \mathbf{X}^{\prime}, t\right)}{\partial t}=\left\{\Delta f_{2}{ }^{D D}\left(\mathbf{X}, \mathbf{X}^{\prime}, t\right)+\Delta^{\prime} f_{2}{ }^{D R}\left(\mathbf{X}, \mathbf{X}^{\prime}, t\right)\right\} H(t) \\
+\delta\left(\mathbf{X}-\mathbf{X}^{\prime}\right) \Delta f_{\mathbf{1}}{ }^{R}(\mathbf{X}) H(t),
\end{gathered}
$$

where

$$
\Delta f^{R}(\mathbf{X}) \equiv \int d^{3} \kappa r_{D}(\boldsymbol{\kappa}) f^{R}(\mathbf{X}, \mathbf{\kappa})=r_{D} \int_{\{\Omega\}} d \Omega f^{R}(\mathbf{X}, \mathbf{\Omega}),
$$

and $r_{D}$ is the detection rate for photons. In Eq. (20) the subscript has been intentionally omitted on the density of photons, designated by $R$, for the sake of generality; and in addition we are now using $f$ to represent densities in the continuous phase space (as contrasted to $F$ in discrete space). We have also invoked the use of a step function $H(t)$ to accompany detection rates; and $\{\boldsymbol{\Omega}\}$ is the set of vectors subtended by the detector as seen by the source. The use of $H(t)$ is a convenient means of accounting for the physical condition that no photons are detected before the detectors are turned on at $t=0$. Recall that we are interpreting experiments of this type to be such that the charged particles arising from detection events are simply recorded and merely accumulate from $t=0$ to $t$, and that all charged-particle (photoelectron) densities and cross densities are assumed to be zero at $t=0$. Therefore it is seen that $D$ densities are continuous functions of time. Their rates (first time derivatives of the densities) are discontinuous at $t=0$. Of course, second time derivatives of $D$ densities are then singular at $t=0$. The equation for the photon singlet is

$$
\begin{aligned}
& {\left[c \boldsymbol{\Omega} \cdot \boldsymbol{\nabla}+r_{s}(\mathbf{X})+\alpha(\mathbf{X})-\boldsymbol{\epsilon}(\mathbf{X})\right] f_{\mathbf{1}}{ }^{R}(\mathbf{X}, \boldsymbol{\Omega})} \\
& =\epsilon(\mathbf{X}, \boldsymbol{\Omega}) \rho_{\boldsymbol{\kappa}}+\int d \Omega^{\prime \prime} \mathfrak{F}^{\mathrm{sc}}\left(\boldsymbol{\Omega}^{\prime \prime} \rightarrow \boldsymbol{\Omega}\right) r_{\boldsymbol{s}}(\mathbf{X}) f_{\mathbf{1}}{ }^{R}\left(\mathbf{X}, \boldsymbol{\Omega}^{\prime \prime}\right),
\end{aligned}
$$

where $r_{s}(\mathbf{X})$ is the photon scattering rate per photon, 
and $\alpha(\mathbf{X})$ and $\epsilon(\mathbf{X})$ are the photon absorption and emission rates, respectively, in the source per photon. For convenience, we have assumed $\alpha$ and $\epsilon$ to be isotropic. The photon scattering frequency, $\mathfrak{F}^{\text {sc }}$ $\left(\boldsymbol{\Omega}^{\prime \prime} \rightarrow \boldsymbol{\Omega}\right) d \Omega$, is the probability that a photon traveling initially in direction $\boldsymbol{\Omega}^{\prime \prime}$, will upon scattering, have a final direction in $d \Omega$ about $\boldsymbol{\Omega}$. Also in these equations $c=3 \times 10^{10} \mathrm{~cm} / \mathrm{sec}$ is the speed of light, as the media are assumed to be nondispersive. Equation (21) for the photon singlet density is recognized to be a Boltzmann equation with an external source, i.e., spontaneous emission. This source is proportional to the source atom density, as are $\alpha$ and $\epsilon$ on the left-hand side of the equation. The photon doublet equation is

$\left[c\left(\boldsymbol{\Omega} \cdot \boldsymbol{\nabla}+\boldsymbol{\Omega}^{\prime} \cdot \boldsymbol{\nabla}^{\prime}\right)+a(\mathbf{X})+r_{s}(\mathbf{X})+a\left(\mathbf{X}^{\prime}\right)+r_{s}\left(\mathbf{X}^{\prime}\right)\right] f_{2}{ }^{R R}\left(\mathbf{X}, \boldsymbol{\Omega} ; \mathbf{X}^{\prime} \mathbf{\Omega}^{\prime}\right)$

where

$$
\begin{aligned}
& =\int d \Omega^{\prime \prime}\left\{\boldsymbol{r}_{s}(\mathbf{X}) \mathfrak{F}^{\mathrm{sc}}\left(\boldsymbol{\Omega}^{\prime \prime} \rightarrow \boldsymbol{\Omega}\right) f_{2}{ }^{R R}\left(\mathbf{X}, \boldsymbol{\Omega}^{\prime \prime} ; \mathbf{X}^{\prime}, \boldsymbol{\Omega}^{\prime}\right)+r_{s}\left(\mathbf{X}^{\prime}\right) \mathfrak{F}^{\mathrm{sc}}\left(\boldsymbol{\Omega}^{\prime \prime} \rightarrow \boldsymbol{\Omega}^{\prime}\right) f_{2}{ }^{R R}\left(\mathbf{X}, \boldsymbol{\Omega} ; \mathbf{X}^{\prime}, \boldsymbol{\Omega}^{\prime \prime}\right)\right\} \\
& +\epsilon(\mathbf{X}, \boldsymbol{\Omega}) \rho_{\kappa} f_{1}{ }_{1}^{R}\left(\mathbf{X}^{\prime}, \mathbf{\Omega}^{\prime}\right)+f_{1}{ }^{R}(\mathbf{X}, \boldsymbol{\Omega}) \epsilon\left(\mathbf{X}^{\prime}, \boldsymbol{\Omega}^{\prime}\right) \rho_{\kappa}+\delta\left(\mathbf{X}-\mathbf{X}^{\prime}\right) H\left(\mathbf{X} ; \boldsymbol{\Omega}, \boldsymbol{\Omega}^{\prime}\right),
\end{aligned}
$$

$$
\begin{aligned}
& H\left(\mathbf{X} ; \boldsymbol{\Omega}, \boldsymbol{\Omega}^{\prime}\right) \equiv \delta\left(\boldsymbol{\Omega}-\boldsymbol{\Omega}^{\prime}\right)\left\{\boldsymbol{\epsilon}(\mathbf{X}, \boldsymbol{\Omega}) \rho_{k}+\left[r_{s}(\mathbf{X})+\alpha(\mathbf{X})+\epsilon(\mathbf{X})\right] f_{\mathbf{1}}{ }^{R}(\mathbf{X}, \boldsymbol{\Omega})\right. \\
& \left.+\int d \Omega^{\prime \prime} r_{s}(\mathbf{X}) \mathscr{F}^{\mathrm{sc}}\left(\boldsymbol{\Omega}^{\prime \prime} \rightarrow \boldsymbol{\Omega}\right) f_{\mathbf{1}}{ }^{R}\left(\mathbf{X}, \boldsymbol{\Omega}^{\prime \prime}\right)\right\}-r_{s}(\mathbf{X}) \mathfrak{F}^{\mathrm{sc}}\left(\boldsymbol{\Omega} \rightarrow \boldsymbol{\Omega}^{\prime}\right) f_{\mathbf{1}}{ }^{R}(\mathbf{X}, \boldsymbol{\Omega}) \\
& -\boldsymbol{r}_{s}\left(\mathbf{X}^{\prime}\right) \mathfrak{F}^{\mathrm{se}}\left(\mathbf{\Omega}^{\prime} \rightarrow \boldsymbol{\Omega}\right) f_{1}{ }^{R}\left(\mathbf{X}^{\prime}, \mathbf{\Omega}^{\prime}\right) .
\end{aligned}
$$

The equation for the cross-doublet density of photons and detected particles is

$$
\begin{aligned}
{\left[\partial / \partial t+c \mathbf{\Omega}^{\prime} \cdot \nabla^{\prime}+r_{s}\left(\mathbf{X}^{\prime}\right)+a\left(\mathbf{X}^{\prime}\right)\right] f_{2}{ }^{D R}\left(\mathbf{X} ; \mathbf{X}^{\prime}, \mathbf{\Omega}^{\prime} ; t\right) } & =\Delta f_{2}{ }^{R}\left(\mathbf{X} ; \mathbf{X}^{\prime}, \mathbf{\Omega}^{\prime}\right) H(t)+\epsilon\left(\mathbf{X}^{\prime}, \mathbf{\Omega}^{\prime}\right) \rho_{\kappa} f_{1}{ }^{D}(\mathbf{X}, t) \\
& +\int d \Omega^{\prime \prime} r_{s}\left(\mathbf{X}^{\prime}\right) F^{\mathrm{sc}}\left(\mathbf{\Omega}^{\prime \prime} \rightarrow \mathbf{\Omega}^{\prime}\right) f_{2}{ }^{D R}\left(\mathbf{X} ; \mathbf{X}^{\prime}, \mathbf{\Omega}^{\prime \prime} ; t\right)-\delta\left(\mathbf{X}-\mathbf{X}^{\prime}\right) r_{D}(\mathbf{X}) f_{1}{ }^{R}(\mathbf{X}, \boldsymbol{\Omega}) H(t) .
\end{aligned}
$$

Note that the equation for $f_{2}{ }^{R D}\left(\mathbf{X}, \boldsymbol{\Omega} ; \mathbf{X}^{\prime} ; t\right)$ is obtained by interchanging arguments. We have also used $a \equiv \alpha-\epsilon$. Recalling that the observables of an occupation-number fluctuation experiment are related to the detected particle variance, it is convenient to obtain the transport equations for the variances. From Eqs. (18)-(24) we find

$$
\begin{array}{r}
\frac{\partial V^{D D}\left(\mathbf{X}, \mathbf{X}^{\prime}, \tau\right)}{\partial \tau}=\left\{\Delta V^{R D}\left(\mathbf{X}, \mathbf{X}^{\prime}, \tau\right)+\Delta^{\prime} V^{D R}\left(\mathbf{X}, \mathbf{X}^{\prime}, \tau\right)\right. \\
\left.+\delta\left(\mathbf{X}-\mathbf{X}^{\prime}\right) \Delta f_{1}{ }^{R}(\mathbf{X})\right\} H(\tau) .
\end{array}
$$

The quantities $V^{R D}\left(\mathbf{X}, \mathbf{X}^{\prime}, \tau\right)$ and $V^{D R}\left(\mathbf{X}, \mathbf{X}^{\prime}, \tau\right)$ are defined as

$$
V^{R D}\left(\mathbf{X}, \mathbf{X}^{\prime}, \tau\right) \equiv f_{2}{ }^{R D}\left(\mathbf{X}, \mathbf{X}^{\prime}, \tau\right)-f_{\mathbf{1}}{ }^{R}(\mathbf{X}) f_{\mathbf{1}}{ }^{D}\left(\mathbf{X}^{\prime}, \tau\right)
$$

and

$$
V^{D R}\left(\mathbf{X}, \mathbf{X}^{\prime}, \tau\right) \equiv f_{2}{ }^{D R}\left(\mathbf{X}, \mathbf{X}^{\prime}, \tau\right)-f_{\mathbf{1}}{ }^{D}(\mathbf{X}, \tau) f_{1}{ }^{R}\left(\mathbf{X}^{\prime}\right) .
$$

The equation for $V^{D R}\left(\mathbf{X} ; \mathbf{X}^{\prime}, \mathbf{\Omega}^{\prime} ; \tau\right)$, as an example, is $\left[\partial / \partial \tau+c \mathbf{\Omega}^{\prime} \cdot \nabla^{\prime}+r_{s}\left(\mathbf{X}^{\prime}\right)+a\left(\mathbf{X}^{\prime}\right)\right] V^{D R}\left(\mathbf{X} ; \mathbf{X}^{\prime}, \mathbf{\Omega}^{\prime} ; \tau\right)$

$$
\begin{aligned}
& =\int d \Omega^{\prime \prime} r_{s}\left(\mathbf{X}^{\prime}\right) F^{\mathrm{se}}\left(\boldsymbol{\Omega}^{\prime \prime} \rightarrow \boldsymbol{\Omega}^{\prime}\right) V^{D R}\left(\mathbf{X} ; \mathbf{X}^{\prime}, \mathbf{\Omega}^{\prime \prime} ; \tau\right) \\
& +\Delta V^{R R}\left(\mathbf{X} ; \mathbf{X}^{\prime}, \boldsymbol{\Omega}^{\prime}\right) H(\tau) \\
& \quad-\delta\left(\mathbf{X}-\mathbf{X}^{\prime}\right) r_{D} f_{\mathbf{1}} R(\mathbf{X}, \boldsymbol{\Omega}) H(\tau) .
\end{aligned}
$$

in like manner

$$
V^{R R}\left(\mathbf{X}, \mathbf{X}^{\prime}\right) \equiv f_{2}{ }^{R R}\left(\mathbf{X}, \mathbf{X}^{\prime}\right)-f_{\mathbf{1}}{ }^{R}(\mathbf{X}) f_{1}{ }^{R}\left(\mathbf{X}^{\prime}\right)
$$

and satisfies the equation

$$
\begin{aligned}
& {\left[c\left(\mathbf{\Omega} \cdot \boldsymbol{\nabla}+\mathbf{\Omega}^{\prime} \cdot \boldsymbol{\nabla}^{\prime}\right)+\boldsymbol{r}_{s}(\mathbf{X})+a(\mathbf{X})+r_{s}\left(\mathbf{X}^{\prime}\right)+a\left(\mathbf{X}^{\prime}\right)\right] V^{R R}\left(\mathbf{X}, \mathbf{\Omega} ; \mathbf{X}^{\prime}, \mathbf{\Omega}^{\prime}\right)} \\
& =\int d \Omega^{\prime \prime}\left[r_{s}(\mathbf{X}) \mathscr{F}^{\mathrm{sc}}\left(\boldsymbol{\Omega}^{\prime \prime} \rightarrow \boldsymbol{\Omega}\right) V^{R R}\left(\mathbf{X}, \boldsymbol{\Omega}^{\prime \prime} ; \mathbf{X}^{\prime}, \boldsymbol{\Omega}^{\prime}\right)\right. \\
& \left.+r_{s}\left(\mathbf{X}^{\prime}\right) \mathfrak{F}^{\mathrm{sc}}\left(\mathbf{\Omega}^{\prime \prime} \rightarrow \boldsymbol{\Omega}^{\prime}\right) V^{R R}\left(\mathbf{X}, \boldsymbol{\Omega} ; \mathbf{X}^{\prime}, \mathbf{\Omega}^{\prime \prime}\right)\right]+\delta\left(\mathbf{X}-\mathbf{X}^{\prime}\right) H\left(\mathbf{X} ; \boldsymbol{\Omega}, \mathbf{\Omega}^{\prime}\right) .
\end{aligned}
$$

The boundary conditions for the variances relating to detected particles are

$$
V^{D D}(0)=V^{D R}(0)=V^{R D}(0)=0 .
$$

Using these boundary conditions, we find by differentiating (25) that

$$
\frac{\partial^{2} V^{D_{1} D_{2}}(\tau)}{\partial \tau^{2}}=\int_{(\text {D.V. })_{1}} d^{3} X_{1} \int_{(\text {D.v. })_{2}} d^{3} X_{2}\left\{\left[\Delta \frac{\partial V^{R D}\left(\mathbf{X}_{1}, \mathbf{X}_{2}, \tau\right)}{\partial \tau}+\Delta^{\prime} \frac{\partial V^{D R}\left(\mathbf{X}_{1}, \mathbf{X}_{2}, \tau\right)}{\partial \tau}\right] H(\tau)+\delta\left(\mathbf{X}_{1}-\mathbf{X}_{2}\right) \Delta f_{1}{ }^{R}\left(\mathbf{X}_{1}\right) \delta(\tau)\right\} .
$$


If we consider a two-detector experiment, the last term of Eq. (32) goes to zero. So we are left only with the task of solving for $V^{R D}$ and $V^{D R}$.

We can formulate a method of solution by considering first the equation for the radiation singlet density. Equation (21) can be written as an integral equation by incorporating an appropriate Green's function as ${ }^{8}$ [letting $\left.\epsilon(\mathbf{X} ; \mathbf{\Omega}) \rho_{\kappa} \equiv S(\mathbf{X}, \mathbf{\Omega})\right]$

$$
f_{\mathbf{1}}^{R}(\mathbf{X}, \mathbf{\Omega})=\int d^{3} X^{\prime} G\left(\mathbf{X}^{\prime}, \mathbf{X}\right) S\left(\mathbf{X}^{\prime}, \mathbf{\Omega}\right)+\int d^{3} X^{\prime} \int d \Omega^{\prime \prime} r_{s}\left(\mathbf{X}^{\prime}\right) \mathfrak{F}^{\mathrm{sc}}\left(\mathbf{\Omega}^{\prime \prime} \rightarrow \mathbf{\Omega}\right) G\left(\mathbf{X}^{\prime}, \mathbf{X}\right) f_{1}{ }^{R}\left(\mathbf{X}^{\prime}, \mathbf{\Omega}^{\prime \prime}\right),
$$

or

where

$$
f_{1}^{R}(\mathbf{X}, \mathbf{\Omega})=I(\mathbf{X}, \mathbf{\Omega})+\Re f_{1}^{R}(\mathbf{X}, \mathbf{\Omega})
$$

$$
I(\mathbf{X}, \mathbf{\Omega}) \equiv \int_{R=0}^{\infty} \frac{d R}{c}\left\{\exp \left[-\int_{R^{\prime}=0}^{R} \frac{d R^{\prime}}{c}\left(r_{s}\left(\mathbf{X}-R^{\prime} \mathbf{\Omega}\right)+a\left(\mathbf{X}-R^{\prime} \mathbf{\Omega}\right)\right)\right]\right\} S(\mathbf{X}-R \mathbf{\Omega}, \mathbf{\Omega}) .
$$

The integral opertor $\mathcal{R}$ is defined as

$$
\Re f_{1}^{R}(\mathbf{X}, \mathbf{\Omega}) \equiv \int d^{3} X^{\prime} \int d \mathbf{\Omega}^{\prime \prime} \int_{p=0}^{\infty} d p\left\{\exp -\left[r_{s}(\mathbf{X})+a(\mathbf{X})\right] p\right\} \delta\left(\mathbf{X}-c p \mathbf{\Omega}-\mathbf{X}^{\prime}\right) r_{s}\left(\mathbf{X}^{\prime}\right) \mathfrak{F}^{\mathrm{sc}}\left(\mathbf{\Omega}^{\prime \prime} \rightarrow \mathbf{\Omega}\right) f_{1}{ }^{R}\left(\mathbf{X}^{\prime}, \mathbf{\Omega}^{\prime \prime}\right)
$$

By successive iterations one gets

$$
f_{1}^{R}(\mathbf{X}, \mathbf{\Omega})=\sum_{i=0}^{\infty} I_{i}(\mathbf{X}, \mathbf{\Omega})
$$

where

and

$$
I_{0}(\mathbf{X}, \mathbf{\Omega})=I(\mathbf{X}, \mathbf{\Omega})=\left[f_{1}^{R}(\mathbf{X}, \mathbf{\Omega})\right]_{0}
$$

$$
I_{i}(\mathbf{X}, \mathbf{\Omega})=\mathcal{R} I_{i-1}(\mathbf{X}, \mathbf{\Omega})=\Re^{i} I_{0}(\mathbf{X}, \mathbf{\Omega}) .
$$

The solution for $V^{R R}$ follows in exactly the same manner. As for $V^{R D}(t)$ and $V^{D R}(t)$, it is equally straightforward but somewhat more tedious to solve the timedependent Boltzmann equation. One just introduces the appropriate time-dependent Green's function.

\section{STATISTICAL PROPERTIES OF A BLACKBODY AND FLUCTUATION MEASUREMENTS THEREOF}

In this section we consider a simple system comprised of only the radiating source and the detectors with their associated circuitry. It is seen that the set of equations describing this system is the same as in the previous section, except that all terms pertaining to photon scattering are deleted. That is, we now have, for just those equations relating to radiation distributions,

$$
\begin{gathered}
{[c \boldsymbol{\Omega} \cdot \boldsymbol{\nabla}+a(\mathbf{X})] f_{1}{ }^{R}(\mathbf{X}, \mathbf{\Omega})=\epsilon(\mathbf{X}, \mathbf{\Omega}) \rho_{\kappa},} \\
{\left[\left(c \mathbf{\Omega} \cdot \boldsymbol{\nabla}+c \mathbf{\Omega}^{\prime} \cdot \nabla^{\prime}\right)+a(\mathbf{X})+a\left(\mathbf{X}^{\prime}\right)\right] V^{R R}\left(\mathbf{X}, \mathbf{\Omega} ; \mathbf{X}^{\prime}, \mathbf{\Omega}^{\prime}\right)} \\
=\delta\left(\mathbf{X}-\mathbf{X}^{\prime}\right) \delta\left(\mathbf{\Omega}-\mathbf{\Omega}^{\prime}\right)\left\{\epsilon(\mathbf{X}, \mathbf{\Omega}) \rho_{\kappa}\right. \\
\left.+[\alpha(\mathbf{X})+\epsilon(\mathbf{X})] f_{1}{ }^{R}(\mathbf{X}, \mathbf{\Omega})\right\} \\
{\left[\partial / \partial t+c \mathbf{\Omega}^{\prime} \cdot \nabla^{\prime}+a\left(\mathbf{X}^{\prime}\right)\right] V^{D R}\left(\mathbf{X} ; \mathbf{X}^{\prime}, \mathbf{\Omega}^{\prime} ; t\right)} \\
=\left\{\Delta V^{R R}\left(\mathbf{X} ; \mathbf{X}^{\prime}, \mathbf{\Omega}^{\prime}\right)-\delta\left(\mathbf{X}-\mathbf{X}^{\prime}\right) r_{D} f_{1}^{R}(\mathbf{X}, \mathbf{\Omega})\right\} H(t)
\end{gathered}
$$

${ }^{8}$ K. M. Case, F. de Hoffmann, and G. Placzek, Introduction to the Theory of Neutron Diffusion (U. S. Government Printing Office, Washington, D. C., 1953). and the equation for $V^{R D}\left(\mathbf{X}, \mathbf{\Omega} ; \mathbf{X}^{\prime} ; t\right)$ is obtained by interchanging arguments of Eq. (42).

At this point let us make a brief digression to consider the statistical properties of just the radiation distribution of a blackbody system. For a system of this type, in which the source atoms are in kinetic equilibrium at a temperature $T$, it can be shown that the radiation emission and absorption rates are related as

where

$$
\alpha(\kappa)=\epsilon(\kappa) \exp \left(\mathcal{E}_{\kappa} / \theta\right),
$$

$$
\begin{gathered}
\mathcal{E}_{\kappa} \equiv \hbar c \kappa, \\
\theta \equiv k T .
\end{gathered}
$$

The equilibrium distribution for the photon singlet density for a sufficiently large homogeneous system is therefore given by

$$
f_{1}^{R}(\boldsymbol{\kappa})=\rho_{\kappa} /\left(e^{\varepsilon_{\kappa} / \theta}-1\right) .
$$

If we define an energy density,

$$
U(\boldsymbol{\kappa}) \equiv \hbar c \kappa f_{1}^{R}(\boldsymbol{\kappa})
$$

we get the conventional expression for blackbody radiation

$$
U(\nu)=\frac{8 \pi h}{c^{3}} \frac{\nu^{3}}{e^{h \nu / \theta}-1}
$$

We have used $\rho_{\kappa}=1 /(2 \pi)^{3}$ and transformed to frequency space according to $2 \pi \nu_{\lambda}=c \kappa$. We also summed over polarizations $\lambda$ and dropped the subscript from $\nu_{\lambda}$. Then solving for the photon variance, analogous to our previous Eq. (30) (except for scattering terms), we get

$V^{R R}\left(\mathbf{X}, \boldsymbol{\kappa} ; \mathbf{X}^{\prime}, \boldsymbol{\kappa}^{\prime}\right)=\delta\left(\mathbf{X}-\mathbf{X}^{\prime}\right) \delta\left(\boldsymbol{\kappa}-\boldsymbol{\kappa}^{\prime}\right)$

$$
\times\left[f_{1}^{R}(\boldsymbol{\kappa}) /\left(1-e^{-\varepsilon_{\boldsymbol{\kappa}} / \theta}\right)\right],
$$

which is in agreement with the result of Landau and Lifshitz. ${ }^{9}$ It is interesting to note from Eq. (48) that the

${ }^{9}$ L. D. Landau and E. M. Lifshitz, Statistical Physics (AddisonWesley Publishing Company, Reading, Massachusetts, 1958). 
blackbody radiation distribution is expected to depart from Poisson only so long as the energy of the radiation that we detect is not significantly greater than $k T$ for the source atom distribution. The implication is clear, therefore, that in principle the effective temperature of the source atoms in appropriate systems may be inferred from the behavior of the fluctuations in the detected particle distribution as a function of the radiation energy that is observed. For more on this topic the reader is referred to Ref. 10, which applies more generally to such a measurement in a dispersivemedium. The possibility of such a temperature measurement was also inferred in Ref. 11.

Turning our attention now to the physical observables of a fluctuation measurement, we deduce expressions for the variance (and/or cross-covariance) of detected particles for three cases (listed as I, II, and III below). The realism of the model in each succeeding case improves as we proceed, and we devote attention to even the very idealized model in Case I because of the widespread discussion that has appeared pertaining to the result that is obtained.

\section{Case I}

Consider an experiment with a single detector that is sensitive to all photon energies in a system that is sufficiently large and homogeneous so that the set of coupled transport equations reduces to

$$
\begin{aligned}
\partial V^{D D}(t) / \partial t & =r_{D}\left\{V^{R D}(t)+V^{D R}(t)+f_{1}{ }^{R}\right\} H(t), \\
{[\partial / \partial t+a] V^{D R} } & =r_{D}\left\{V^{R R}-f_{1}^{R}\right\} H(t), \\
V^{R R} & =\left(1 / \rho_{\kappa}\right)\left(f_{1}\right)^{2}+f_{1}{ }^{R}, \\
f_{1}^{R} & =\epsilon \rho_{\kappa} / a,
\end{aligned}
$$

Solving Eq. (50) and substituting the result into Eq. (49), which is then integrated from $t=0$ to $t=T_{0}$, we get

$$
V^{D D}\left(T_{0}\right)=\left\langle N_{T_{0}}{ }^{D}\right\rangle\left[1+\frac{\left\langle N_{T_{0}}^{D}\right\rangle}{\gamma T_{0}}\right] \text { for } T_{0} \gg 1 / a,
$$

where $^{12}$

and

$$
\gamma \equiv a \rho_{\kappa} / 2=(\alpha-\epsilon) / 16 \pi^{3}
$$

$$
\left\langle N_{T_{0}}{ }^{D}\right\rangle \equiv r_{D} f_{1}{ }^{R} T_{0} .
$$

Of course formula (53) is recognized to correspond to the result obtained by Purcell. ${ }^{13,14}$ More will be said on

${ }^{10}$ R. K. Osborn and A. Z. Akcasu, University of Michigan Technical Report administered through Office of Research Administration, ORA Project 07599, 1966 (unpublished).

${ }^{11}$ R. Hanbury Brown and R. Q. Twiss, Proc. Roy. Soc. 243A, pg. 291 (1957) Eq. (2.20).

${ }^{12}$ If the atomic states have finite lifetimes it can be shown that $a$ in Eq. (54) is proportional to the spectral bandwidth for a given line of radiation, so long as observation times are long compared to the lifetimes of the emitting and absorbing states. Indeed it is evident in the present analysis (from the explicit calculation of expressions for $\alpha$ and $\epsilon$ ) that $\gamma^{-1}$ is the lifetime associated with the emitting and absorbing atomic states.

${ }_{13}$ E. M. Purcell, Nature 178, 1449 (1956).

${ }^{14}$ McCombie (Ref. 16) has also presented an analogous calculation. the observability of this effect at later points in our discussion.

\section{Case II}

In this case let us consider a spatially dependent, finite experiment in which two distinct point detectors view the radiating system from separate observation points ( $\mathbf{X}$ and $\mathbf{X}^{\prime}$ with $\left.\left|\mathbf{X}-\mathbf{X}^{\prime}\right|<T_{0} / c\right)$ in a vacuum outside the radiating source. If a photon is observed by one detector, it is not available for detection by the other detector. In this case, Eqs. (40)-(42) are readily solved and one finds $V^{D D}\left(\mathbf{X}, \mathbf{X}^{\prime}, T_{0}\right)=0$ unless the extension of the line joining $\mathbf{X}$ and $\mathbf{X}^{\prime}$ extends into some region of the radiating source. It has also been assumed that not every photon incident upon the first detector is necessarily absorbed by it. If these conditions are fulfilled one finds

$$
V^{D D}\left(\mathbf{X}, \mathbf{X}^{\prime}, T_{0}\right) \propto\left\langle N_{T_{0}}{ }^{D}\right\rangle \frac{1}{e^{\varepsilon_{\kappa} / \theta}-1} .
$$

Here again the implications are apparent regarding the possibility of measuring the temperature of the system from a fluctuation measurement (see Ref. 10 for a more general consideration of the details of such a measurement). In this case the detectors are assumed to be sensitive only to a limited range of photon energies about $\mathcal{E}_{\kappa}$, and $\mathbf{X}$ and $\mathbf{X}^{\prime}$ are assumed to be "on the same side" of the radiation source (that is, the source cannot lie between the two detectors). Aside from the trivial conclusion that both detectors must "see" the source, this result is still not very startling-for, one notes that when considering photons about the phase point $\left(\mathbf{X}^{\prime \prime}, \mathbf{k}\right)$ (in the source), the probability for the emission of a photon at $\left(\mathbf{X}^{\prime \prime}, \boldsymbol{r}\right)$ is enhanced due to the dependence of the transition probability per unit time, $T_{e}\left(\boldsymbol{\kappa} \mid \mathbf{K}_{1}, a_{1}\right.$; $\left.\mathbf{K}_{2}, a_{2}\right)$ \{proportional to $\left[1+\rho^{R}\left(\mathbf{X}^{\prime \prime}, \mathbf{k}\right)\right]$ \} upon the photon occupation number in the final state. It is of particular importance to recall here that, due to the uncertainty principle, we have (at the outset) coarse-grained $\mu$ space into six-dimensional phase cells of volume $h^{3}$ or greater such that the coordinate $\mathbf{X}^{\prime \prime}$ merely locates the center of a three-dimensional cell in configuration space that is compatible with the momentum uncertainty implied by the spectral bandwidth of the radiation line that is being observed. Thus the origin of this correlation effect is explicitly seen to arise from the Bose-Einstein statistical properties of the radiation emission process within a single phase cell. ${ }^{15}$ The effect is potentially observable when both detectors can "see" photons with a given direction vector having their origin in a common phase cell. It is seen that the first term of Eq. (3), $\left\langle N_{T_{0}}{ }^{D}\right\rangle$, does not appear in Eq. (56) [and subsequently

15 This effect is best observed in simple chaotic source systems in the absence of strong modal effects. Modal effects or other processes may tend to change the over-all statistical properties of the total system, such that the simple Bose-Einstein statistical features that obtain for the radiation, per se, may become unobservable. Such is the case, for instance, for a single mode of a. laser operating well above threshold. 
in Eq. (57)] because, by using two distinct detectors that physically occupy different phase cells, the "selfcorrelated" random noise associated with just the detectors is eliminated. It should be noted that the notion of the phase cell has not entered the present analysis in an ad hoc or intuitive manner, but is rather a fundamental concept as indicated above. Its role is central to a theory that attempts a simultaneous description of the spatial and momentum dependence of physically observable quantities, such as distribution functions that are associated in practice with measurements on finite, inhomogeneous systems.

\section{Case III}

Finally we consider a situation very much like Case II, but now using more realistic detectors of finite, nonzero volume. In this case the variance is nonzero only if the detectors are aligned within a distance $T_{0} / c$ of each other, one behind the other, along a line of sight extending into the radiation source. Furthermore at least some portion of each detector must commonly share one or more lines of sight extending into the source. We then get

$$
V^{D_{1} D_{2}}\left(T_{0}\right) \propto \frac{\left\langle N_{T_{0}}{ }^{D}\right\rangle^{2}}{\gamma T_{0}} A_{1} \bigcap A_{2},
$$

where $A_{1} \bigcap A_{2}$ represents the effective area of "overlap" between the two detectors (noting that one is behind the other) as viewed from the radiating source. Of course, in the limit that these finite detectors approach point detectors, one can show that the results of Eqs. (56) and (57) become identical. This result as well as the final remarks of Case II above are recognized to constitute a generalization of the work of Purcell ${ }^{13}$ and $\mathrm{McCombie}^{16}$ in which an infinite homogeneous model was implied, corresponding to Case I above. The difference lies in the fact that the present treatment, which specifically accounts for photon streaming and other finite, spatially dependent effects, now gives specific information regarding the geometric requirements for seeing the "photon bunching" effect. In Appendix A we will analyze an example suggested by Purcell, i.e., to consider a two-detector experiment in which the initial beam of radiation from a chaotic optical source is split by a polarizer. The observable quantity is the cross-covariance of detected particles from photocathodes placed in the resultant beams of radiation with orthogonal polarizations. While the result is well known, the present approach accounts for the macroscopic result from a deductive point of view that explicitly exhibits the microscopic origin of the effect. This effect is seen to be a direct consequence of the quantum selection rules that obtain for the com-

${ }^{16} \mathrm{C}$. W. McCombie, in Fluctuation, Relaxation, and Resonance in Magnetic Systems, edited by D. Ter Haar (Plenum Press, Inc., New York, 1962). puted expressions for the emission (and absorption) transition rates that appear in the kinetic equations.

\section{ON RADIATION-DETECTOR-ATOM COUPLING}

It can finally be noted that our kinetic theoretic formalism has been developed with the retention of sufficient statistical generality in the generic equations [Eqs. (8) and (10)] to account for effects such as the coupling between the radiation and detector atom distributions. Thus the present theory can be systematically applied to examine, in explicit terms, the extent to which the incident-radiation distribution may affect the distribution of detector atoms. In a great part of the literature dealing with radiation measurements, "weak coupling" is assumed. That is, the detector atom distribution is assumed to be known and to be independent of the incident radiation impinging upon the detector. We have also made this assumption in Secs. IV and V [although the generic equations (8) and (10) are perfectly general in this respect], inasmuch as our major effort has been to develop a kinetic theory appropriate to the analysis of fluctuations in radiation distributions in finite, inhomogeneous systems. ${ }^{17}$

To recognize the point at which our work departs from numerous other approaches in which the "weakcoupling" approximation is made, it is well to recall the operational means by which this approximation was invoked in the respective cases. Representative of much of the existing work in this area, Kelley and Kleiner ${ }^{18}$ have demonstrated in considerable detail that the "weak-coupling" assumption allows the assertion that the output of a photodetector is proportional to the instantaneous intensity of the incident radiation field. The utility of being able to make this assertion lies in the fact that correlations in the detector outputs can be simply stated in terms of just the field correlation functions whether viewed classically or quantum mechanically. Therefore, using the work of Kelley and Kleiner ${ }^{18}$ as a convenient point of reference, it is seen that they accomplish the "weak-coupling" approximation by a factorization on the density matrix, per se, as in their Eq. (4.11). Once this is done the possibility of deductively accounting for higher-order statistical coupling is, of course, lost. On the other hand, the density matrix employed in deriving the generic kinetic equations for multiplet densities herein is the density matrix for the total system. Approximations such as the "weak-coupling" approximation are accomplished by later reductions on the generalized multiplet densities, which are the variables, or observables, of the theory. Therefore the restrictions imposed by this approximation procedure are readily relaxed in the present

${ }^{17}$ In view of this "weak-coupling" specialization, our results for the illustrative examples of Secs. IV and V, except for the spatially dependent generalizations, are equivalent to the results obtained from other points of view (see Refs. 13, 16, 20).

18 P. L. Kelley and W. H. Kleiner, Phys. Rev. 136, A316 (1964). 
case, and the generality of our basic theory is not impaired in any fundamental way. A truncation of the multiplet densities at a given statistical order can then be systematically evaluated by relaxing that reduction to the next higher statistical order. The price paid for retaining multiplet densities of higher and higher statistical order is that a progressively larger set of coupled equations must be solved. But the additional equations, in any case, are all of the same mathematical form as the lower-order equations; so the difficulties associated with generalization here are problems in accounting and not in concept. Specifically, it can be recalled that, in Sec. IV, we specialized our set of equations to obtain the "weak-coupling" approximation (between the radiation and detector atoms) by letting

$$
F_{2}{ }^{A R}=F_{1} A F_{1}{ }^{R} \text {. }
$$

This factorization asserts that $V^{A R}=0$, which is tantamount to assuming that the detector atom distribution $F_{1}{ }^{A}$ is known in principle, independent of the radiation field. To examine the implications of this specialization (to the next higher statistical order), the above factorization is not assumed, and terms proportional to $V^{A R}$ are retained in the existing equations as well as now including additional equations for $V^{A R}$ and for $V^{D A}(t)$, as we shall see. Thus the following set of equations is sufficiently general to account for radiation and detector atom coupling to lowest statistical order (we are considering an infinite homogeneous system here only for the sake of convenience to the present illustrative example; in general one can account for finite effects with no basic difficulty, as well).

$$
\partial F_{1}{ }^{D}(t) / \partial t=M_{D} F_{1}{ }^{A} F_{1}{ }^{R} H(t)+\left\{M_{D} V^{A R} H(t)\right\}
$$

$$
\begin{gathered}
\partial V^{D D}(t) / \partial t=2 M_{D} F_{1}^{A} V^{D R}(t)+M_{D} F_{1}^{A} F_{1}{ }^{R} H(t) \\
+\left\{\left[2 M_{D} F_{1}^{R} V^{A D}+M_{D} V^{A R}\right] H(t)\right\} \\
{\left[\partial / \partial t+r_{a}\right] V^{D R}(t)=r_{D}\left[V^{R R}-F_{1}{ }^{R}\right] H(t)} \\
+\left\{M_{D}\left[F_{1}{ }^{R}-1\right] V^{A R} H(t)-M_{D} F_{1}{ }^{A} V^{A D}(t)\right\} \\
r_{a} F_{1}^{R}=\epsilon \rho_{\mathrm{k}}-\left\{M_{D} V^{A R}\right\} \\
r_{a} V^{R R}=r_{a} F_{1}^{R}+\epsilon F_{1}^{R} \\
-\left\{M_{D}\left[F_{1}{ }^{R}-1\right] V^{A R}\right\}
\end{gathered}
$$

where

$$
\begin{aligned}
r_{D} & =M_{D} F_{1}{ }^{A}, \\
r_{a} & =a+r_{D} .
\end{aligned}
$$

We have invoked the prescription of Ron ${ }^{19}$ to truncate triplet densities in this set of equations as

$F_{3}{ }^{B C D}=F_{1}{ }^{B} F_{1}{ }^{C} F_{1}{ }^{D}+F_{1}{ }^{B} V^{C D}+F_{1}{ }^{C} V^{B D}+F_{1}{ }^{D} V^{B C}$.

It is also noted that the quantity $r_{a}$ replaces the quantity denoted as $a$ in our equations in previous sections. It is therefore apparent that Eqs. (58)-(62)

${ }^{19}$ A. Ron, J. Math. Phys. 4, 1182 (1963). are strict analogs of Eqs. (49)-(52) that apply to the illustrative example in Sec. V-the only difference being that terms arising due to the retention of radiationdetector-atom coupling appear in \{\} in the present instance. Accordingly it is clear from a comparison of the structure of these two sets of equations that the conventional result of Purcell ${ }^{13}$ and many others ${ }^{16,20}$ [see Eq. (53)] is now extended and takes on a more general form (that is dependent upon $V^{A R}$, among other factors) by the above inclusion of coupling effects. Of course it is necessary to deduce equations of motion for $F_{1}{ }^{A}, V^{A D}(t)$, and $V^{A R}$ in order to obtain order-ofmagnitude estimates of the relevance of these coupling effects. Deduction of these additional equations of motion from Eqs. (8) and (10) is entirely straightforward, but it is found that the parameters entering these equations are distinctive of particular physical features of individual experimental systems of interest. To proceed further from this point, devoting adequate attention to the sizeable array of details that arise, is a sizeable, although tractable, program worthy of separate consideration. Such an extended program is not crucial to (or, in fact, compatible with) the present development of the basic elements of a spatially dependent kinetic theory of higher statistical order for radiating systems and is therefore deferred to a later report.

\section{DISCUSSION}

The main objective of this paper has been to present a physically interpretable space- and momentumdependent kinetic theory to higher statistical order (than the first) for radiating systems. Incorporating the statistical axioms of quantum mechanics and using the Liouville equation to express the dynamical axiom, equations of motion for observable variables are deduced in rather general terms. These variables are chosen to be expectation values of first- and second(and, if necessary, higher) order moments of number densities of particles and photons comprising a given system. It is an elemental part of the development that the simultaneous specification of the space and momentum dependence of these multiplet densities is compatible with, and, in fact, limited fundamentally by the uncertainty principle. This limitation is accounted for formally by coarse graining the six-dimensional phase space into hypercells of volume $h^{3}$ or greater. It can be realized that the phase cell concept is basic to the foundations of any physical theory attempting a spaceand momentum-dependent description of finite, inhomogeneous systems. It becomes particularly important in radiating systems because the extent of the three-dimensional portion of the phase cell in configuration space may be of macroscopic proportion for the radiation. Thus the distribution functions that we are dealing with are to be interpreted in a coarse-grained sense. In contrast, the analogous distribution functions,

${ }^{20}$ L. Mandel and E. Wolf, Rev. Mod. Phys. 37, 231 (1965). 
discussed from a classical point of view, must be interpreted in the limit of vanishing $d^{3} x d^{3} p$. Since this is not physically meaningful, the approach taken herein is felt to be a favorable alternative. Our treatment therefore has been quantum mechanical, incorporating the statistical axioms of quantum mechanics to define expectation values, and using the quantum Liouville equation to express the behavior of the joint probability density for the entire system. Further specializations are achieved by approximations on expectation values of number operators (multiplet densities) and not on the density matrix, per se. This is of particular importance for maintaining statistical generality so that explicit account may be taken of coupling effects as discussed in Sec. VI. It has been our intent to examine from a tractable, deductive kinetic theoretic point of view relevant portions of the underlying basis upon which the study of stochastic processes may be exploited to infer information pertinent to the dynamic behavior of an interacting system by passive observations on the emergent radiation distributions. In order to be able, ultimately, to propose and suitably interpret measurements on more complicated systems we have devoted appreciable commentary to the importance of an appropriate recognition and definition of the distinguishing characteristics of the actual observables and of the dominant physical features of individual experimental situations.

\section{ACKNOWLEDGMENTS}

The authors are indebted to Professor A. Z. Akcasu for his stimulating assistance throughout the course of this investigation. We also gratefully acknowledge private communication with Professor Kenneth $\mathbf{M}$. Watson.

\section{APPENDIX A: THE EFFECT OF A POLARIZING BEAM SPLITTER ON A FLUCTUATION MEASUREMENT}

Let us now consider the system illustrated by Fig. 1. We have let $S(\mathbf{X}, \mathbf{\Omega})$ be the image of a filtered, chaotic mercury arc source focused on an aperture having

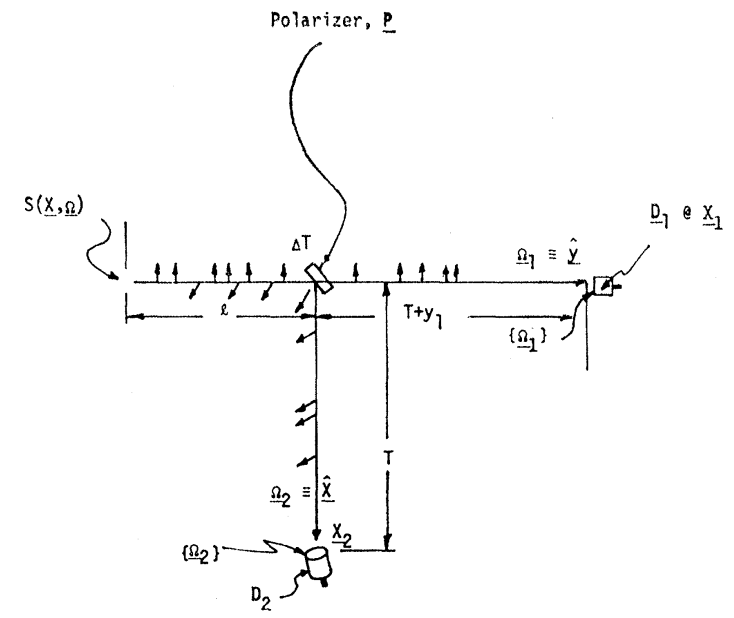

FIG. 1. Line drawing of a fluctuation experiment including a polarizer to split the initial beam. $y_{1}$ accounts for any difference in path lengths.

dimensions much greater than the mean wavelength of the radiation. We are now considering a system with a polarizer $\mathbf{P}$ present in the path of the beam emitted from the secondary source $S(\mathbf{X}, \mathbf{\Omega})$. Thus photons from the initial beam that have a given polarization represented by $\uparrow,\left(\lambda=\lambda_{1}\right)$, are transmitted to detector $D_{1}$. Photons having the orthogonal polarization (represented by $\downarrow, \lambda=\lambda_{2}$ ) are scattered to detector $D_{2}$. We assume that the radiation source is such that photons having the above polarizations are distributed randomly in the initial beam impinging upon $\mathbf{P}$. It is apparent here that the function of the polarizer is essentially that of a photon scatterer. We therefore consider that the photons (with polarization $\lambda=\lambda_{1}$ ) in the beam impingent upon detector $D_{1}$ have been simply transmitted by the polarizer. Photons in the scattered beam (with polarization $\lambda=\lambda_{2}$ ) that impinges upon detector $D_{2}$ are assumed, of course, to have been scattered by the polarizer. It now remains only to solve the appropriate set of equations, using the iteration technique described by Eqs. (33)-(39). The set of working equations for this case is written as (we cannot sum over photon polarizations here):

$$
[c \boldsymbol{\Omega} \cdot \boldsymbol{\nabla}+A(\mathbf{X}, \lambda)] f_{\mathbf{1}}^{R}(\mathbf{X}, \boldsymbol{\Omega}, \lambda)=\epsilon(\mathbf{X}, \mathbf{\Omega}, \lambda) \rho_{\kappa}+\int d \Omega^{\prime \prime} r_{S}\left(\mathbf{X}, \lambda^{\prime \prime}\right) \mathfrak{F}^{\mathrm{sc}}\left(\mathbf{\Omega}^{\prime \prime}, \lambda^{\prime \prime} \rightarrow \mathbf{\Omega}, \lambda\right) f_{\mathbf{1}}{ }^{R}\left(\mathbf{X}, \mathbf{\Omega}^{\prime \prime}, \lambda^{\prime \prime}\right)
$$

where

$$
A(\mathbf{X}, \lambda) \equiv r_{S}(\mathbf{X}, \lambda)+a(\mathbf{X}, \lambda)
$$

$\left[c\left(\mathbf{\Omega} \cdot \boldsymbol{\nabla}+\mathbf{\Omega}^{\prime} \cdot \boldsymbol{\nabla}^{\prime}\right)+A(\mathbf{X}, \lambda)+A\left(\mathbf{X}^{\prime}, \lambda^{\prime}\right)\right] V^{R R}\left(\mathbf{X}, \mathbf{\Omega}, \lambda ; \mathbf{X}^{\prime}, \mathbf{\Omega}^{\prime}, \lambda^{\prime}\right)$

$$
\begin{aligned}
=\int d \Omega^{\prime \prime}\left\{r_{S}\left(\mathbf{X}, \lambda^{\prime \prime}\right) F^{\mathrm{se}}\left(\mathbf{\Omega}^{\prime \prime}, \lambda^{\prime \prime} \rightarrow \mathbf{\Omega}, \lambda\right) V^{R R}\left(\mathbf{X}, \mathbf{\Omega}^{\prime \prime}, \lambda^{\prime \prime} ; \mathbf{X}^{\prime}, \mathbf{\Omega}^{\prime}, \lambda^{\prime}\right)+r_{S}\left(\mathbf{X}^{\prime}, \lambda^{\prime \prime}\right)\right. \\
\left.\quad \times F^{\mathrm{se}}\left(\mathbf{\Omega}^{\prime \prime}, \lambda^{\prime \prime} \rightarrow \mathbf{\Omega}^{\prime}, \lambda\right) V^{R R}\left(\mathbf{X}, \mathbf{\Omega}, \lambda \mathbf{X}^{\prime}, \mathbf{\Omega}^{\prime \prime}, \lambda^{\prime \prime}\right)\right\}+\delta\left(\mathbf{X}-\mathbf{X}^{\prime}\right) H\left(\mathbf{X} ; \boldsymbol{\Omega}, \lambda ; \mathbf{\Omega}^{\prime}, \lambda^{\prime}\right)
\end{aligned}
$$


where

$$
\begin{aligned}
& H\left(\mathbf{X} ; \boldsymbol{\Omega}, \lambda ; \mathbf{\Omega}^{\prime}, \lambda^{\prime}\right)=\delta\left(\boldsymbol{\Omega}-\mathbf{\Omega}^{\prime}\right) \delta_{\lambda \lambda^{\prime}}\left\{\epsilon(\mathbf{X}, \boldsymbol{\Omega}, \lambda) \rho_{\kappa}+[A(\mathbf{X}, \lambda)+2 \epsilon(\mathbf{X}, \lambda)]\right. \\
& \left.\times f_{\mathbf{1}}{ }^{R}(\mathbf{X}, \mathbf{\Omega}, \lambda)+\int d \mathbf{\Omega}^{\prime \prime} r_{\mathbf{S}}\left(\mathbf{X}, \lambda^{\prime \prime}\right) \mathcal{F}^{\mathrm{sc}}\left(\mathbf{\Omega}^{\prime \prime}, \lambda^{\prime \prime} \rightarrow \mathbf{\Omega}, \lambda\right) f_{\mathbf{1}} R\left(\mathbf{X}, \mathbf{\Omega}^{\prime \prime}, \lambda^{\prime \prime}\right)\right\} \\
& -r_{S}(\mathbf{X}, \lambda) \mathscr{F}^{\mathrm{sc}}\left(\boldsymbol{\Omega}, \lambda \rightarrow \boldsymbol{\Omega}^{\prime}, \lambda^{\prime}\right) f_{\mathbf{1}}{ }^{R}(\mathbf{X}, \boldsymbol{\Omega}, \lambda)-r_{S}\left(\mathbf{X}^{\prime}, \lambda^{\prime}\right) F^{\mathrm{sc}}\left(\mathbf{\Omega}^{\prime}, \lambda^{\prime} \rightarrow \boldsymbol{\Omega}, \lambda\right) f_{\mathbf{1}}{ }^{R}\left(\mathbf{X}^{\prime}, \mathbf{\Omega}^{\prime}, \lambda^{\prime}\right)
\end{aligned}
$$

Also

$$
\begin{array}{r}
{[\partial / \partial t+c \mathbf{\Omega} \cdot \boldsymbol{\nabla}+A(\mathbf{X}, \lambda)] V^{R D}\left(\mathbf{X}, \boldsymbol{\Omega}, \lambda ; \mathbf{X}^{\prime} ; t\right)=r_{D}\left(\mathbf{X}^{\prime}\right)\left[\int d \Omega^{\prime} V^{R R}\left(\mathbf{X}, \mathbf{\Omega}, \lambda ; \mathbf{X}^{\prime}, \mathbf{\Omega}^{\prime}, \lambda^{\prime}\right)-\delta\left(\mathbf{X}-\mathbf{X}^{\prime}\right) f_{\mathbf{1}}^{R}\left(\mathbf{X}^{\prime}, \mathbf{\Omega}^{\prime}, \lambda^{\prime}\right)\right] H(t)} \\
+\int d \Omega^{\prime \prime} r_{S}\left(\mathbf{X}, \lambda^{\prime \prime}\right) \mathcal{F}^{\mathrm{sc}}\left(\mathbf{\Omega}^{\prime \prime}, \lambda^{\prime \prime} \rightarrow \mathbf{\Omega}, \lambda\right) V^{R D}\left(\mathbf{X}, \mathbf{\Omega}^{\prime \prime}, \lambda^{\prime \prime} ; \mathbf{X}^{\prime} ; t\right)
\end{array}
$$

The equation for $V^{D R}\left(\mathbf{X} ; \mathbf{X}^{\prime}, \mathbf{\Omega}^{\prime}, \lambda^{\prime} ; t\right)$ is obtained by interchanging the primed and unprimed arguments. Noting that the polarizer is very localized in space we can write

$$
\begin{aligned}
& r_{S}\left(\mathbf{X}_{j}, \lambda_{j}\right)=r_{S}\left\{H\left[(l+\Delta T) \mathbf{\Omega}_{1}-\mathbf{X}_{j}\right]-H\left(l \mathbf{\Omega}_{1}-\mathbf{X}_{j}\right)\right\} \\
& \text { for } \lambda_{j}=\lambda_{2} \\
& =0 \quad \text { for } \lambda_{j}=\lambda_{1} \text {, }
\end{aligned}
$$

where $H$ is again the unit step function and $\Delta T$ is the thickness of the polarizer. In the limit that $\Delta T$ approaches zero, we have

$$
\begin{aligned}
& \lim _{\Delta T \rightarrow 0}(1 / \Delta T)\left\{H\left[(l+\Delta T) \mathbf{\Omega}_{1}-\mathbf{X}_{j}\right]\right. \\
& \text { and } \\
& \left.-H\left(l \mathbf{\Omega}_{1}-\mathbf{X}_{j}\right)\right\} \rightarrow \delta\left(\mathbf{X}_{j}-l \mathbf{\Omega}_{1}\right)
\end{aligned}
$$

The angular dependence for a photon-scattering event is given by

$$
\begin{aligned}
& \mathfrak{F}^{\mathrm{sc}}\left(\boldsymbol{\Omega}, \lambda \rightarrow \mathbf{\Omega}_{2}, \lambda_{2}\right)=F^{\mathrm{sc}}\left(\boldsymbol{\Omega}_{1} \rightarrow \mathbf{\Omega}_{2}\right) \delta\left(\mathbf{\Omega}_{1}-\mathbf{\Omega}\right) \delta_{\lambda \lambda_{2}}, \\
& \mathfrak{F}^{\mathrm{sc}}\left(\boldsymbol{\Omega}, \lambda \rightarrow \mathbf{\Omega}_{1}, \lambda_{1}\right)=0 .
\end{aligned}
$$

If we assume that a photon scatters but once (at most) at the polarizer, only one iteration is needed to obtain an exact solution of our set of working equations. We then get

$$
\begin{aligned}
f_{1}^{R}\left(\mathbf{X}_{1}, \mathbf{\Omega}_{1}, \lambda_{1}\right) & =\epsilon\left(\mathbf{\Omega}_{1}, \lambda_{1}\right) \rho_{\kappa}\left[1-e\left\{-\frac{a\left(\lambda_{1}\right) d}{c}\right\}\right], \\
f_{1} R\left(\mathbf{X}_{1}, \mathbf{\Omega}_{1}, \lambda_{2}\right) & =0 \\
f_{1}^{R}\left(\mathbf{X}_{2}, \mathbf{\Omega}_{2}, \lambda_{1}\right) & =0 \\
f_{1}{ }^{R}\left(\mathbf{X}_{2}, \mathbf{\Omega}_{2}, \lambda_{2}\right) & =F^{\mathrm{sc}}\left(\mathbf{\Omega}_{1} \rightarrow \mathbf{\Omega}_{2}\right) \boldsymbol{\epsilon}\left(\mathbf{\Omega}_{1}, \lambda_{2}\right) \rho_{\kappa} \\
\times & {\left[1-e\left\{-\frac{a\left(\lambda_{2}\right) d}{c}\right\}\right] . }
\end{aligned}
$$

We have used the physical condition that a photon with polarization $\lambda_{2}$ impinging upon the polarizer is scattered with certainty and appears in the reflected beam (without being absorbed by the polarizer). We have also assumed that the photon absorption and emission rates, $\alpha$ and $\epsilon$, are constant within the source, and that the effective source thickness is $d$ for photons traveling in $d \Omega_{1}$ about $\mathbf{\Omega}_{\mathbf{1}}$. Solving for $V^{R D}\left(\mathbf{X}_{\mathbf{1}}, \mathbf{\Omega}_{1}, \lambda_{\mathbf{1}} ; \mathbf{X}_{2}, t\right)$ and $V^{D R}\left(\mathbf{X}_{1} ; \mathbf{X}_{2}, \mathbf{\Omega}_{2}, \lambda_{2} ; t\right)$, we find after some substitution and manipulation, that

$$
V^{D_{1} D_{2}}\left(T_{0}\right)=0 .
$$

This result from the present deductive approach is in agreement with the statement of Purcell ${ }^{13}$ and others ${ }^{20,21}$ that orthogonal components of randomly polarized (unpolarized) radiation are uncorrelated.

${ }^{21}$ W. Martienssen and E. Spiller, Phys. Rev. Letters 16, 531 (1966). 